\title{
AIP | Physics of

\section{A fast model for spreading of neutral particles injected locally into hot plasma}

\section{Z. Tokar}

Citation: Physics of Plasmas 21, 082517 (2014); doi: 10.1063/1.4894100

View online: http://dx.doi.org/10.1063/1.4894100

View Table of Contents: http://scitation.aip.org/content/aip/journal/pop/21/8?ver=pdfcov

Published by the AIP Publishing

\section{Articles you may be interested in}

A coarse-grained transport model for neutral particles in turbulent plasmas

Phys. Plasmas 19, 122310 (2012); 10.1063/1.4771688

Modeling of non-stationary local response on impurity penetration in plasma

Phys. Plasmas 19, 042502 (2012); 10.1063/1.3701555

Turbulent and neoclassical impurity transport in tokamak plasmas

Phys. Plasmas 16, 032306 (2009); 10.1063/1.3083299

Fluid-particle hybrid simulation on the transports of plasma, recycling neutrals, and carbon impurities in the Korea Superconducting Tokamak Advanced Research divertor region

Phys. Plasmas 12, 062504 (2005); 10.1063/1.1914807

Modeling of beryllium deposition from a beryllium-seeded deuterium plasma

J. Appl. Phys. 97, 064912 (2005); 10.1063/1.1862309

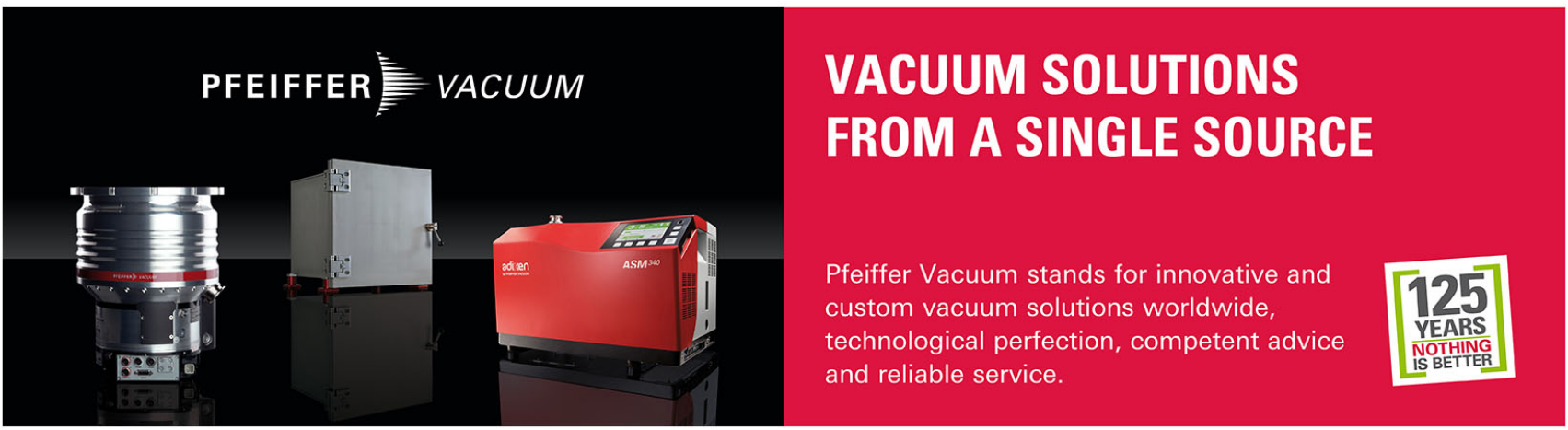




\title{
A fast model for spreading of neutral particles injected locally into hot plasma
}

\author{
M. Z. Tokar \\ Institut für Energie- und Klimaforschung - Plasmaphysik, Forschungszentrum Jülich GmbH, \\ Trilateral Euregio Cluster, D-52425 Jülich, Germany
}

(Received 17 April 2014; accepted 4 August 2014; published online 29 August 2014)

A fast model for calculation of non-stationary 3-D profiles of the density for neutral particles locally released into a hot plasma is elaborated. The approach reduces non-stationary three-dimensional transport equations to a set of one-dimensional ones describing the time evolution of the radial profiles for several parameters characterizing adequately the three-dimensional structure. The method is applied to model the spreading process of carbon atoms released by laser desorption in an experimental device and the local injection of working gas into a fusion reactor. The associated heat loads onto the first wall are assessed. (C) 2014 AIP Publishing LLC.

[http://dx.doi.org/10.1063/1.4894100]

\section{INTRODUCTION}

Under diverse circumstances, neutral particles, both of working hydrogen isotopes and of impurities, can enter very locally the plasma of fusion devices. This is the case, e.g., by the usage of laser-based diagnostics, such as laser-induced desorption spectroscopy (LIDS) and laser-induced ablation spectroscopy (LIAS), applied to measure and monitor the retention of hydrogen and impurities in the wall components. ${ }^{1}$ By a short intense pulse of laser radiation onto a tiny spot on a plasma facing component (PFC), performed during a plasma discharge, particles of hydrogen isotopes and impurities, deposited in the PFC are released into the plasma. Here, they are excited and ionized by electrons and, by measuring the intensity of their line radiation, one can judge about the amount of particles emitted from the wall and its composition. Also, the injection of the working deuterium gas into the plasma applied for the density control and other means is normally done through valves with the inlet area much smaller than the wall surface. Calculations of the density of released particles, predicting its variation in time and space, are of significant interest for the interpretation of experimental results.

Diverse processes, involving injected neutral particles, can lead to significant disturbances in the plasma. Thus, the energy spent by electrons on the particle excitation results in the reduction of the temperature, the delivery of electrons by the ionization leads to the increase in the density. ${ }^{2}$ Since the plasma parameters are essentially involved into interpretation of measurements, one has to assess the perturbations induced by the injected neutral particles. Such an assessment also requires the information about the spatial profiles of the neutral density.

Routinely, Monte Carlo statistical approaches are applied to treat the behavior of neutral particles in fusion plasmas, see, e.g., Refs. 3 and 4. However, these methods require large CPU time and are too tedious to be used under conditions of very fast changing in time and threedimensionally inhomogeneous densities of neutrals. In this paper, we propose a more primitive but faster approach, allowing to assess roughly the maximum density and characteristic dimensions of the regions occupied by particles on the flux surface as functions of time and the minor radius of the surface. It includes integration of several non-stationary one-dimensional partial differential equations. The approach developed is demonstrated by calculating the radiation pattern of carbon atoms released from the wall in LIAS experiments in tokamak TEXTOR $^{1}$ and by estimating the heat loads onto the first wall in the fusion reactor ITER induced by a local puffing of the working gas. The remainder of the paper is organized as follows. In Sec. II, non-stationary three-dimensional transport equations for primary neutrals released into the plasma and for secondary atoms generated there by collisions of primary ones with the background ions are formulated. In Sec. III, an approach to find approximate solutions of these equations is outlined. Examples of applications are given in Sec. IV and final conclusions are drawn in Sec. V.

\section{BASIC EQUATIONS}

The present approach is based on the division of neutral species in two groups. The former one includes particles, called, henceforth, "primary" species, which are released directly from the wall with velocities defined by the injection mechanism. For any mechanism, their radial velocities, i.e., along the minor radius $r$ perpendicular to the flux surfaces, are directed towards the plasma core. Primary species are ionized by electrons in the plasma and their velocity can be changed in collisions with ions. These interactions can be of elastic and inelastic nature, as in the case of hydrogen atoms for which resonant charge-exchange is the dominant process of such a type. We assume that collisions with ions lead to the "vanishing" of primary species and generation of "secondary" ones with the velocity and temperature of the ions. Since the latter is magnetized, their averaged velocity perpendicular to the magnetic field is much smaller than the thermal one. Therefore, the perpendicular velocities of secondary neutrals are directed nearly randomly and their transport can be modeled in a fluid approximation. In the toroidal 
geometry of fusion devices, cylindrical coordinate systems with the axes along radial, poloidal, and toroidal directions are normally used. The penetration depth of neutral particles entering the plasma from a wall element is, however, significantly smaller than the plasma dimensions. Therefore, by considering the spreading of particles in question, we operate in an orthogonal coordinate system with the origin at the injection spot on the wall, $r=r_{w}$, with the axes $x=r_{w}-r$, directed towards the plasma core, and $y$ and $l$, oriented perpendicular and parallel to field lines, respectively, at the magnetic surface touching the wall, $x=0$.

\section{A. Primary neutrals}

The velocity distribution functions of primary particles with respect to the velocity components in the directions $y$ and $l$ are identical. Thus, additionally to $V_{x}$, it is sufficient to take into account the velocity distribution with respect to the component $V_{\rho}$ in a cylindrical reference system with $\varrho=\sqrt{x^{2}+l^{2}}$. Consider primary particles released from the wall with velocities in infinitesimally small ranges $d V_{x}$ and $d V_{\rho}$ in the vicinity of the values $V_{x}$ and $V_{\rho}$. At the wall, the fraction of such particles in the total density $n_{0}$ of primary neutrals is $f\left(V_{x}, V_{\rho}\right) d V_{x} d V_{\rho}$, where the distribution function $f\left(V_{x}, V_{\rho}\right)$ is assumed as a Maxwellian one with respect to $V_{\rho}$ and a one-side Maxwellian one shifted with the drift velocity $V_{m}$, for $V_{x}^{4}$

$$
\begin{aligned}
f\left(V_{x} \geq 0, V_{\rho} \geq 0\right)= & \frac{4 V_{\rho}}{\sqrt{\pi}\left[1+\operatorname{erf}\left(V_{m} / V_{t h}\right)\right] V_{t h}^{3}} \\
& \times \exp \left[-\frac{\left(V_{x}-V_{m}\right)^{2}+V_{\rho}^{2}}{V_{t h}^{2}}\right] .
\end{aligned}
$$

Here, $\operatorname{erf}\left(V_{m} / V_{t h}\right)$ is the probability integral, $V_{t h}=\sqrt{2 T_{0} / m}$, with $T_{0}$ and $m$ being the temperature and mass of primary particle, and the form-factor arises due to the normalization $\int_{0}^{\infty} d V_{x} \int_{0}^{\infty} f\left(V_{x}, V_{\rho}\right) d V_{\rho}=1$. The density of primary species in question, $d n_{0}\left(V_{x}, V_{\rho}\right)$, changes in time and space according to the following continuity equation:

$$
\partial_{t} d n_{0}+V_{x} \partial_{x} d n_{0}+\left(V_{\rho} / \rho\right) \times \partial_{\rho}\left(\rho d n_{0}\right)=-\nu d n_{0},
$$

where $\nu=\nu_{i o n}+\nu_{*}$ is the total "loss" frequency, $\nu_{i o n}=$ $k_{i o n}^{0} n_{e}$ the ionization frequency, with $k_{i o n}^{0}$ being the ionization rate coefficient and $n_{e}$ the electron density; $\nu *$ is the frequency of collisions with background ions leading to the generation of secondary particles; $\nu_{*}=k_{c x} n_{i}$ for atoms of hydrogen isotopes, with $k_{c x}$ being the charge-exchange rate coefficient and $n_{i}$ the density of ions. Equation (2) is solved for all $V_{x}$ and $V_{\rho}$ and the total density of primary particles is calculated as $n_{0}(t, x, \rho)=\int d n_{0}\left(V_{x}, V_{\rho}\right)$.

\section{B. Secondary neutrals}

The total density of the primary neutral loss, $\nu * n_{0}$, gives the source density for secondary particles and their density $n *$ is governed by the continuity equation

$$
\partial_{t} n_{*}+\vec{\nabla} \cdot \vec{\Gamma}=\nu_{*} n_{0}-\nu_{i o n} n_{*},
$$

where $\vec{\Gamma}$ is their particle flux density. As it was mentioned above, the mass velocity of secondary particles is small compared with the thermal velocity and their momentum transport can be described in a fluid approximation by neglecting inertia term

$$
\vec{\nabla}\left(\frac{p_{*}}{m}\right) \approx \nu_{*}\left(n_{0}+n_{*}\right) \vec{V}_{i}-\nu \vec{\Gamma} .
$$

Here, $p_{*}=n_{*} T_{*}$ is the pressure of secondary neutrals; only the parallel component $V_{l}$ of the ion mass velocity $\vec{V}_{i}$ may be of interest in Eq. (4). Thus, this provides

$$
\Gamma_{x, y}=-d_{*} \partial_{x, y} p_{*} ; \quad \Gamma_{l}=-d_{*} \partial_{l} p_{*}+u_{*} p_{*}+u_{l} \nu_{*} n_{0}
$$

with $d_{*}=1 /(m \nu), u_{l}=V_{l} / \nu$, and $u_{*}=u_{l} \nu_{*} / T_{*}$. The temperature $T *$ of secondary neutrals can be assessed from a heat balance equation

$$
\partial_{t} p_{*}+\nabla \cdot\left(\vec{\Gamma} T_{*}\right)=\nu_{*}\left(n_{0}+n_{*}\right) T_{i}-\nu p_{*},
$$

where the heating of the secondary species by collisions with the ions towards their temperature $T_{i}$ is taken into account. Thus, collisions with ions provide for primary species an additional to ionization particle loss channel, see the last term in the right hand side (r.h.s.) in Eq. (2). The latter results in the source for secondary particles, their parallel momentum and energy, see the first terms in the r.h.s. of Eqs. (3), (4), and (6). As a result, the parallel velocity and temperature of secondary neutrals approach to those of ions.

Finally, consider validity of certain approximations done in the model. Assess possible consequences of the magnetic shear $\hat{s} \equiv d \ln q / d \ln r$, where $q$ is the safety factor. Due to this, the direction $l$ does not coincide everywhere with that of field lines and on magnetic surfaces at $x>0, V_{l}$ is smaller than the actual ion parallel velocity $V_{\|}$. The difference between $V_{\|}$and $V_{l}$ is of $\Delta V_{\|}=V_{\|}[1-\cos (\Delta \psi)]$, where $\Delta \psi \approx l_{*} \hat{S} /(q R)$ with $l_{*}$ being the radial penetration depth of secondary neutrals and $R$ the major radius of the surface. By assuming roughly $l_{*} \simeq 0.03 \mathrm{~m}, \hat{s} \simeq q$, and $R \simeq 1 \mathrm{~m}$, we get $\Delta V_{\|} \simeq V_{\|}(\Delta \psi)^{2} \simeq 10^{-3} V_{\|}$, i.e., the shear effect on the transport of neutrals in the $l$-direction is negligible. The fraction $V_{y}=V_{\|} \sin (\Delta \psi)$ of $V_{\|}$is oriented in the $y$-direction and can affect this component of the neutral transfer. However, $V_{y} \ll$ $V_{\|}$and the effect of $V_{y}$ can be also neglected. Moreover, we consider in the present paper the situation with neutrals released into the plasma far from limiter or divertor target plates. Here, $V_{\|}$is much smaller than the ion sound velocity $c_{s}=\sqrt{2 T_{i} / m}$ (Ref. 5) and this justifies the omission of the inertia term in Eq. (4). Even for neutrals, entering the plasma very close to the target plates, where $V_{\|}$is comparable with $c_{s}$, the omitted inertia term does not prevail over that due to the pressure gradient, because the neutral mass velocity and temperature approach to those of the ions simultaneously.

\section{APPROACH TO SOLUTION}

Numerical integration of non-stationary two/threedimensional partial differential equations is a CPU time demanding procedure. Here, we reduce these equations to a 
set of one-dimensional ones for the time evolution of the radial profiles for several characteristic parameters, namely, the maximum density and characteristic dimensions of the region on the magnetic surface where particles in question are mostly localized.

\section{A. Primary particles}

For these species, the following approximate form of the density profile is assumed:

$$
d n_{0}(t, x, \rho)=d \eta_{0}(t, x) \exp \left[-\frac{\rho^{2}}{\lambda^{2}(t, x)}\right],
$$

which logically mimics the fact that on each magnetic surface, the density of primary particles is localized in some vicinity of the ejection position. This is a reasonable approximation since due to disintegration processes, primary particles are absent far enough from their source. As the next step, we introduce two new dependent variables

$$
\begin{aligned}
& d N_{0}(t, x) \equiv \int_{0}^{\infty} d n_{0} 2 \pi \rho d \rho=\pi \lambda^{2} d \eta_{0} \\
& d \Lambda_{0}(t, x)=\int_{0}^{\infty} d n_{0} 2 \pi \rho^{2} d \rho=\frac{\sqrt{\pi}}{2} \lambda d N_{0} .
\end{aligned}
$$

Equations for $d N_{0}(t, x)$ and $d \Lambda_{0}(t, x)$ follow by the integration of Eq. (2) with respect to $\rho$ with the weights $2 \pi \rho$ and $2 \pi \rho^{2}$, respectively,

$$
\begin{aligned}
\partial_{t} d N_{0}+V_{x} \partial_{x} d N_{0} & =-\nu d N_{0}, \\
\partial_{t} d \Lambda_{0}+V_{x} \partial_{x} d \Lambda_{0} & =V_{\rho} d N_{0}-\nu d \Lambda_{0} .
\end{aligned}
$$

Obviously, the former of Eq. (9) can be derived independently from the assumption (7) on the $\rho$-dependence of the solution and describes the evolution with $t$ and $r$ of the amount of primary particles in question on the magnetic surface per unit length in the direction $r$. Equations (9) can be straightforwardly integrated numerically by discretizing time and spatial derivatives with the steps $\tau$ and $h=x_{i+1}-x_{i}$, respectively,

$$
\begin{aligned}
& \partial_{t} d N_{0}(t, x)=\left(d N_{0, i}^{t}-d N_{0, i}^{t-\tau}\right) / \tau, \\
& \partial_{x} d N_{0}(t, x)=\left(d N_{0, i}^{t}-d N_{0, i-1}^{t}\right) / h,
\end{aligned}
$$

where $i$ counts the spatial grid points $x_{i}$. This approach provides absolutely stable recurrent relations, see Ref. 6

$$
\begin{aligned}
d N_{0, i}^{t} & =\frac{d N_{0, i}^{t-\tau}+\tau d N_{0, i-1}^{t} V_{x} / h}{1+\tau\left(V_{x} / h+\nu\right)} \\
d \Lambda_{0, i}^{t} & =\frac{d \Lambda_{0, i}^{t-\tau}+\tau\left(d \Lambda_{0, i-1}^{t} V_{x} / h+d N_{0, i}^{t} V_{\rho}\right)}{1+\tau\left(V_{x} / h+\nu\right)} .
\end{aligned}
$$

To realize them, one needs initial conditions and boundary conditions at $x_{1}=0$. For those, we consider two cases of particular interest: (i) Particles are injected very locally and brusquely into the plasma, e.g., by laser desorption, during a time by orders of magnitude shorter than a typical time step $\tau$ in our calculations. Nonetheless, the latter has to be chosen so that the distance $s_{x}=\tau V_{x}$ covered by particles in the radial direction during one time step is significantly smaller than their free path before collisions with the background electrons and ions. In such a case, the actual initial radial profile of $d n_{0}$ is of no importance. Here, we assume that at $t=0$, all particles fill homogeneously the fist sell $0 \leq x \leq h$, but practically the same results are obtained for other assumptions, e.g., for $d n_{0}$ decaying exponentially with $s_{x}$ adopted as the $e$-folding length; along the wall, the source region is localized in the irradiated spot with the radius $\rho_{0}$. For the variables $d N_{0}$ and $d \Lambda_{0}$, this results in the following initial conditions:

$$
\begin{aligned}
& d N_{0}(0, x \leq h)=\frac{N_{t o t}}{h} f\left(V_{x}, V_{\rho}\right) d V_{x} d V_{\rho} \\
& d N_{0}(0, x>h)=0 ; \quad d \Lambda_{0}(0, x)=\frac{\sqrt{\pi}}{2} \rho_{0} d N_{0}(0, x),
\end{aligned}
$$

where $N_{\text {tot }}$ is the total amount of injected particles. No particles are released from the wall element after the irradiation pulse and $N(t, 0)=\Lambda(t, 0)=0$ is the boundary condition at $t>0$.

(ii) Particles are entering the plasma quasi-steadily through the area $S_{i n j}$ with the total influx $\Phi_{0}(t)$, e.g., by a gas puff. In this case, the boundary conditions for $N$ and $\Lambda$ are

$$
\begin{aligned}
& d N_{0}(t, 0)=\frac{\Phi_{0}}{\left\langle V_{x}\right\rangle} f\left(V_{x}, V_{\rho}\right) d V_{x} d V_{\rho}, \\
& d \Lambda_{0}(t, 0)=0.5 \sqrt{S_{\text {inj }}} d N_{0}(t, 0),
\end{aligned}
$$

where $\left\langle V_{x}\right\rangle=V_{m}+\frac{V_{t h}}{\sqrt{\pi}} \frac{\exp \left(-V_{m}^{2} / V_{t h}^{2}\right)}{1+e r f\left(V_{m} / V_{t h}\right)}$ is the mean radial velocity of particles; the initial conditions are $d N_{0}(0, x)$ $=d \Lambda_{0}(0, x)=0$.

With known $d N_{0}(t, x)$ and $d \Lambda_{0}(t, x)$, one can obtain the original parameters characterizing the local density of neutrals

$$
d \eta_{0}(t, x)=\frac{\left(d N_{0}\right)^{3}}{\left(d \Lambda_{0}\right)^{2}}, \quad \lambda(t, x)=\frac{2 d \Lambda_{0}}{\sqrt{\pi} d N_{0}} .
$$

The $\rho$-profile of the total density of primary particles is approximated analogously to the relation (7)

$$
n_{0}(t, x, \rho)=\eta_{0}(t, x) \exp \left(-\frac{\rho^{2}}{\lambda_{0}^{2}(t, x)}\right),
$$

where the maximum value

$$
\eta_{0}(t, x)=\int d \eta_{0}\left(V_{x}, V_{\rho}\right),
$$

and the radius of the localization region is defined by conserving the total number of particles on the magnetic surface, $N_{0}(t, x)=\int d N_{0}\left(V_{x}, V_{\rho}\right)$ : 


$$
\lambda_{0}(t, x)=\sqrt{\frac{N_{0}(t, x)}{\pi \eta_{0}(t, x)}} .
$$

\section{B. Secondary neutrals}

Equation (3), with the flux density components given by Eq. (5), involves the parameter $u_{l}$ related to the parallel velocity of the background ions. A parallel flow of the main plasma may be caused by diverse factors not related to the local injection of neutral species. ${ }^{7}$ This contribution is characterized by the value $\bar{u}_{l}$ assumed constant in the whole region where neutrals are present. In addition, localized injection can itself trigger plasma motion. Indeed, by injection of the working gas, the ions, generated by the ionization of neutrals, spread from the injection position. ${ }^{8}$ If the injection rate of either main or impurity neutrals is, however, so high that the local plasma is cooled down through the energy losses, the decrease in the pressure induced by the temperature drop triggers a motion towards the injection position. ${ }^{9}$ In the absence of a mean plasma flow, $\bar{u}_{l}=0$, the motion induced by the injection is symmetric with respect to the position of the neutral entrance, i.e., its velocity has to be zero at $l=0$; the "stagnation point" is, however, shifted if $\bar{u}_{l} \neq 0$. These features can be roughly taken into account by assuming

$$
u_{l}=\bar{u}_{l}(t, x)+\hat{u}_{l}(t, x) \times \tanh \left[\frac{l-l_{m}(t, x)}{l_{*}(t, x)}\right],
$$

where the characteristic dimension of the region affected by injected neutrals, $l_{*}$, and the shift of the "stagnation point," $l_{m}$, have to be determined from the neutral model itself. An approximate solution to Eq. (3), considered as an equation for $p_{*}$, is searched for in the following form:

$$
p_{*}(t, x, y, l)=\omega_{*}(t, x) \times \exp \left(-\frac{y^{2}}{\lambda_{*}^{2}(t, x)}-\frac{\left[l-l_{m}(t, x)\right]^{2}}{l_{*}^{2}(t, x)}\right) .
$$

To find the parameters $\omega_{*}, \lambda_{*}, l_{*}$, and $l_{m}$, we introduce new dependent variables

$$
\begin{aligned}
& P_{*}(t, x) \equiv \int_{-\infty}^{\infty} d y \int_{-\infty}^{\infty} p_{*} d l=\pi \lambda_{*} l_{*} \omega_{*}, \\
& \Delta_{*}(t, x) \equiv \int_{-\infty}^{\infty} y^{2} d y \int_{-\infty}^{\infty} p_{*} d l=P_{*} \frac{\lambda_{*}^{2}}{2}, \\
& \Lambda_{*}(t, x) \equiv \int_{-\infty}^{\infty} d y \int_{-\infty}^{\infty} p_{*} l d l=P_{*} l_{m}, \\
& \Pi_{*}(t, x) \equiv \int_{-\infty}^{\infty} d y \int_{-\infty}^{\infty} n_{*} l^{2} d l=P_{*}\left(\frac{l_{*}^{2}}{2}+l_{m}^{2}\right)
\end{aligned}
$$

explicitly related to the original ones

$$
\begin{gathered}
\omega_{*}=\frac{P_{*}}{\pi \lambda_{*} l_{*}}, \quad \lambda_{*}=\sqrt{\frac{2 \Delta_{*}}{P_{*}}}, \quad l_{*}=\sqrt{2\left(\frac{\Pi_{*}}{P_{*}}-\frac{\Lambda_{*}^{2}}{P_{*}^{2}}\right)}, \\
l_{m}=\frac{\Lambda_{*}}{P_{*}} .
\end{gathered}
$$

The corresponding integrals of (3) provide equations for the new variables

$$
\begin{gathered}
\partial_{t} \frac{P_{*}}{T_{*}}-\partial_{x}\left(d_{*} \partial_{x} P_{*}\right)=\pi \lambda_{0}^{2} \nu_{*} \eta_{0}-\nu_{\text {ion }} \frac{P_{*}}{T_{*}} \\
\partial_{t} \frac{\Lambda_{*}}{T_{*}}-\partial_{x}\left(d_{*} \partial_{x} \Lambda_{*}\right)=\pi \lambda_{0}^{3} \nu_{*} \eta_{0}\left(\bar{u}_{l}-\frac{l_{m} \hat{u}_{l}}{l_{m}+l_{*}}\right) \\
+\bar{u}_{l} \nu_{*} \frac{P_{*}}{T_{*}}-\nu_{\text {ion }} \frac{\Lambda_{*}}{T_{*}} \\
\partial_{t} \frac{\Delta_{*}}{T_{*}}-\partial_{x}\left(d_{*} \partial_{x} \Delta_{*}\right)= \\
\partial_{t} \frac{\lambda_{*}^{4}}{\Pi_{*}} \nu_{*} \partial_{x}\left(d_{*} \partial_{x} \Pi_{*}\right)= \\
+\pi \frac{\lambda_{0}^{4}}{2} \nu_{*} \eta_{0}\left(1+\frac{16}{\sqrt{\pi}} \frac{\hat{u}_{l}}{l_{m}+l_{*}}\right) \\
+2 d_{*} P_{*}+\bar{u}_{l} \nu_{*}\left(0.726 l_{*} \frac{P_{*}}{T_{*}}+2 \frac{\Lambda_{*}}{T_{*}}\right) \\
-\nu_{\text {ion }} \frac{\Pi_{*}}{T_{*}} .
\end{gathered}
$$

For fixed $t$ and $x$, the temperature of secondary neutrals is assumed constant over their whole localization area. By combining Eq. (17) and the integral of Eq. (6) weighted by 1 , we get the following equation for $T_{*}(t, x)$ :

$$
\partial_{t} T_{*}-\frac{T_{*}}{P_{*}} d_{*} \partial_{x} P_{*} \partial_{x} T_{*}=\nu_{*}\left(1+\frac{\pi \lambda_{0}^{2} \eta_{0} T_{*}}{P_{*}}\right)\left(T_{i}-T_{*}\right) .
$$

By discretizing the time derivatives, one reduces Eqs. (17)-(21) to ordinary differential ones. The initial conditions at $t=0$ to Eqs. (17)-(20) are $P_{*}, \Delta_{*}, \Lambda_{*}, \Pi_{*}=0$, and $T_{*}=T_{i}$ to Eq. (21). At the plasma axis, $r=0$ or $x=r_{w}$, the radial derivative of the pressure reduces to zero and, thus, $\partial_{x} P_{*}$, $\partial_{x} \Delta_{*}, \partial_{x} \Lambda_{*}, \partial_{x} \Pi_{*}=0$. At the wall, $x=0$, we take into account that particles, escaping from the plasma, can return back due to recycling with some probability $R_{\text {rec }}<1$. This can be formalized as $n_{*}^{+}=R_{\text {rec }} n_{*}^{-}$, where $n_{*}^{+}$and $n_{*}^{-}$are the densities of secondary particles, moving in the directions of positive and negative $x$, respectively. Since both particle groups have velocities of the thermal one, $V_{*}=\sqrt{2 T_{*} /(\pi m)}$, the boundary condition above relates the pressure $p_{*}=\left(n_{*}^{+}+n_{*}^{-}\right) T_{*}$ and flux density $-d_{*} \partial_{x} p_{*}=\left(n_{*}^{+}-n_{*}^{-}\right) V_{*}$ of the secondary atoms

$$
\frac{1-R_{\text {rec }}}{1+R_{\text {rec }}} p_{*}=\frac{d_{*} T_{*}}{V_{*}} \partial_{x} p_{*} .
$$

Similar relations hold for the variables $P_{*}, \Delta_{*}, \Lambda_{*}$, and $\Pi_{*}$. Since Eq. (21) is of the first order, one boundary condition is only required, which, however, should not contradict to the equation itself. Secondary neutrals spread from their source region, where primary species are localized, in directions towards both the plasma boundary and core. Therefore, the $x$-component of their flux and, thus, $\partial_{x} P_{*}$ reduce to zero at 
some position $x_{*}$. By taking this into account and discretizing the time derivative, we have from Eq. (21) an algebraic equation for $T_{*}^{t}=T_{*}\left(t, x_{*}\right)$ :

$$
\frac{T_{*}^{t}-T_{*}^{t-\tau}}{\tau}=\nu_{*}\left(1+\frac{\pi \lambda_{0}^{2} \eta_{0} T_{*}^{t}}{P_{*}}\right)\left(T_{i}-T_{*}^{t}\right) .
$$

With found $T_{*}\left(t, x_{*}\right)$, Eq. (21) is integrated in both directions from $x_{*}$ to find $T_{*}\left(t, x \lessgtr x_{*}\right)$. Equations (17)-(21) are coupled non-linearly and have to be solved by iterations. The non-linearity is, however, weak and already the third iteration gives a very small error.

\section{APPLICATIONS OF THE MODEL}

\section{A. Laser ablation of carbon}

The release of particles by laser radiation is used, in particular, in LIAS-diagnostics to study the wall composition in fusion devices. ${ }^{1}$ The laser pulse and particle release are very short in this case and the initial conditions are described by the relations (11). In the TEXTOR tokamak, the laser radiation has been concentrated on a spot on the wall with an area of $0.15 \mathrm{~cm}^{-2}$, corresponding to $\rho_{0} \approx 0.22 \mathrm{~cm}$, and typically $N_{\text {tot }} \approx 10^{17}$ of $\mathrm{C}$ atoms were released per pulse. Time of flight measurements can be well interpreted by the velocity distribution (1) with $V_{t h} \approx 5 \mathrm{~km} \mathrm{~s}^{-1}$ and $V_{m} \approx 8.7 \mathrm{~km} \mathrm{~s}^{-1}$. The radial profiles of the plasma density $n_{e}$ and electron temperature $T_{e}$ in TEXTOR Ohmic discharges with the LIAS on carbon samples are shown in Figure 1. In these experiments, the radiation emitted in the $y$-direction due to the transition $2 s^{2} 2 p 3 p^{3} P \rightarrow 2 s^{2} 2 p 3 s^{3} P$ in carbon atoms with the wave length of $909.8 \mathrm{~nm}$ has been recorded. Below, the rate of emitted photon is assessed by assuming that the level $2 s^{2} 2 p 3 p^{3} P$ is populated by the excitation from the ground state $2 s^{2} 2 p^{2}{ }^{3} P$ and spontaneously decays through the transition in question

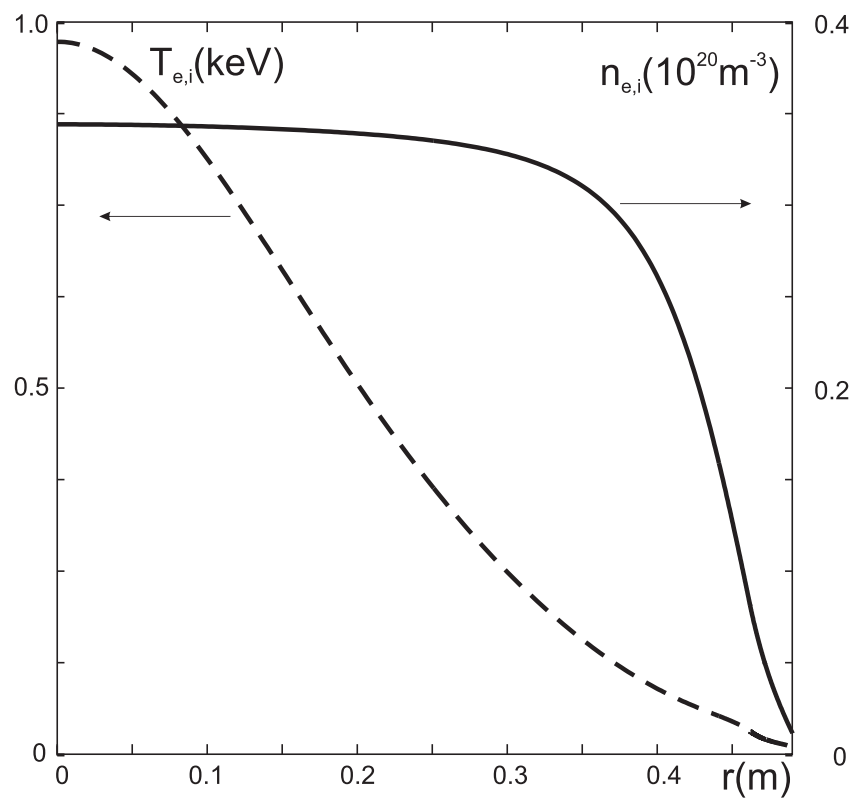

FIG. 1. Radial profiles of the parameters in Ohmic plasma in TEXTOR used in calculations for spreading of carbon atoms.

$$
N_{p h}=\int_{-\infty}^{\infty} k_{e x} n_{e} n_{0} d y=k_{e x} n_{e} \eta_{0} \sqrt{\pi} \lambda_{0} \exp \left(-l^{2} / \lambda_{0}^{2}\right)
$$

where the excitation rate coefficient $k_{e x}\left(T_{e}\right)$ from the ground state $2 s^{2} 2 p^{2}{ }^{3} P$ to the level $2 s^{2} 2 p 3 p^{3} P$ has been calculated by using data from Ref. 10; the infinite integration limits mimic the fact that the neutral penetration depth is much smaller than the plasma dimensions. Figure 2 shows the $N_{p h}(r=r, l)$ computed for different times $t$ after the laser pulse. Although absolute measurements are still not reliable enough to do a detailed quantitative comparison with the results of calculations, picture 2 reproduces well the main qualitative features of the experimental observations: ${ }^{11}$ the maximum of radiation, located at time $t=1 \mu \mathrm{s}$ in the direct vicinity of the irradiated spot on the wall, is shifted by later time moments to the last closed flux surface (LCFS) at $r=0.465 \mathrm{~m}$ defined by the limiter; the toroidal and radial extensions of the radiation cloud rise up to $0.04 \mathrm{~m}$; the radiation of carbon atoms decays during a characteristic time of several $\mu \mathrm{s}$. This is in a rough agreement with primitive estimates of the impurity ionization time as $t_{\text {ion }} \sim 1 / \nu_{\text {ion }}$ $=1 /\left(k_{i o n}^{0} n_{e}\right)$. For the plasma parameters at the LCFS, $n_{e} \approx 10^{19} \mathrm{~m}^{-3}$ and $T_{e} \approx 50 \mathrm{eV}$, we get $k_{\text {ion }}^{0} \approx 10^{-13} \mathrm{~m}^{3} \mathrm{~s}^{-1}$ and $t_{\text {ion }} \approx 1 \mu \mathrm{s}$. This qualitative agreement with observations and simple estimates gives a hope that the cost in accuracy one has to pay for the reduction of 3-D equations to $1-\mathrm{D}$ ones is not too large to overweight the tremendous reduction of the CPU time, roughly by a factor of $10^{4} .{ }^{12}$ In the calculations above, it was assumed that the plasma parameters in the neutral cloud are the same as before the LIAS application. However, as it was demonstrated in our previous calculations, see Ref. 2, under stationary conditions, an influx of carbon atoms of a much low intensity can lead to a significant cooling of the plasma in the neutral impurity cloud through the energy losses on excitation and ionization of impurity particles. Moreover, the supply of electrons by the ionization results in the increase in the local electron density. Such a modification of the local plasma requires a certain time, see non-stationary calculations for lithium injection in Ref. 9. Therefore, it is not straightforward that a shot impurity influx induced by a nanosecond laser pulse also noticeably affects the plasma. This point will be investigated elsewhere.

\section{B. Puffing of the working gas}

Such a puffing is foreseen, e.g., in ITER for diverse purposes. Also in this case, the neutral density will be strongly enhanced locally on the flux surfaces in the vicinity of the injection position. Both the energy losses due to radiation of excited neutrals and the outflow of hot atoms generated by charge-exchange can lead to intensive local heat loads onto the wall and to its damage. If such puff positions will be located inside the ports used for impurity studies, the hydrogen radiation can disturb noticeably spectroscopic measurements. By providing estimates for the local neutral density, the approach outlined above allows to evaluate the relevant parameters. The puffed gas of hydrogen isotopes consists of 

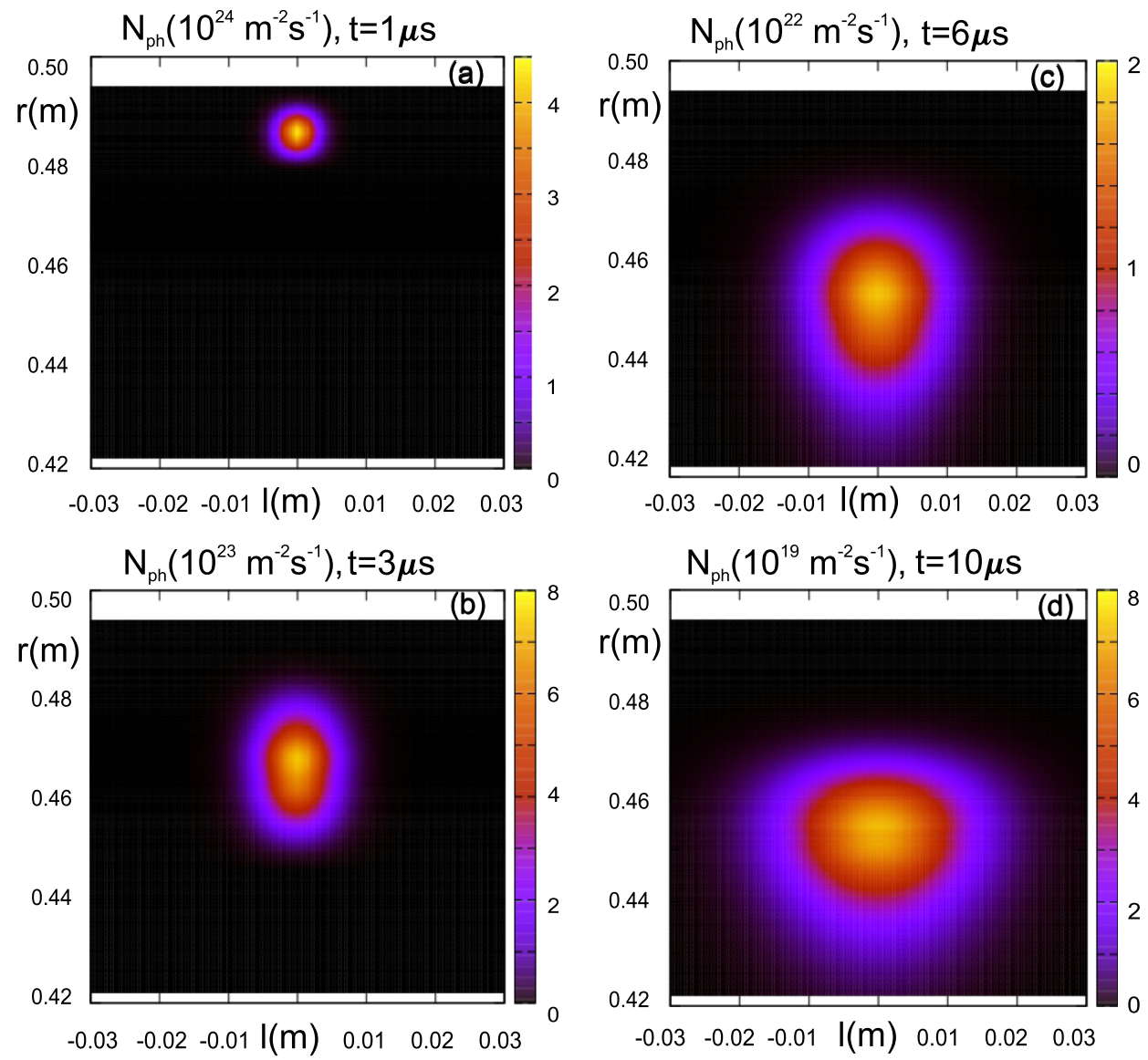

FIG. 2. The rate of photons with the wave length of $909,8 \mathrm{~nm}$ emitted by carbon atoms at different times after the laser pulse: $1 \mu \mathrm{s}$ (a), $3 \mu \mathrm{s}$ (b), $6 \mu \mathrm{s}$ (c), and $10 \mu s$ (d) molecules. Nonetheless, we assume that there is a quasisteady influx of primary atoms with the effective temperature of $2 \mathrm{eV}$ generated by the dissociation of molecules. This is motivated by the fact that the penetration depth of slow molecules is very short, of $0.001 \mathrm{~m}$. One can include molecules into the consideration by necessity, similarly to the approach applied for atoms. Below we consider a situation without plasma flow along the magnetic field, i.e., $\bar{u}_{l}$ and $\hat{u}_{l}$ are zero in Eqs. (18) and (20). Due to the azimuthal symmetry, the energy load onto the wall, $x=0$, approaches its maximum at the puffing axis, $y=l=0$. For the density of the radiation flux, one gets $q_{\text {rad }}^{w}=\int_{0}^{\infty} d x \int_{-\infty}^{\infty} F_{r a d}^{w} d l$, where

$$
F_{\text {rad }}^{w}(x, l)=\int_{-\infty}^{\infty} d y \frac{L_{\text {rad }} n_{e}\left(n_{0}+n_{*}\right)}{4 \pi\left(x^{2}+y^{2}+l^{2}\right)} \cos \psi
$$

with $L_{\text {rad }}$ being the cooling rate of hydrogen atoms whose dependence on the electron density and temperature is taken from Ref. 13, $\psi$ the angle between the direction from the emission point $(x, y, l)$ to the wall point in question, $(0,0,0)$, and the normal to the wall; $\cos \psi=x / \sqrt{x^{2}+y^{2}+l^{2}}$. With $\lambda_{*}=l_{*}$ one gets

$$
q_{\text {rad }}^{w}=0.5 \int_{0}^{\infty} L_{r a d} n_{e}\left[\eta_{0} \sigma\left(\frac{x}{\lambda_{0}}\right)+\eta_{*} \sigma\left(\frac{x}{\lambda_{*}}\right)\right] d x,
$$

where $\sigma(\xi)=1-\sqrt{\pi} \xi \exp \left(\xi^{2}\right)[1-\operatorname{erf}(\xi)]$.

By estimating the energy flux transported by hot atoms, one has to take into account that in the edge transport barrier (ETB) and divertor scrape-off layer (SOL), the mean free path of these particles may be comparable with the characteristic length for the temperature change in the radial direction. Therefore, hot atoms with energies significantly higher than the ion temperature at $x=0$ may escape onto the wall. Even if the fraction of such particles is small in the total outflow of charge-exchanged atoms, they can be of extreme importance, e.g., for sputtering of the wall if $T_{i}(x=0)$ is close or below the sputtering threshold energy. Atoms, generated through charge-exchange in the plasma elementary volume with coordinates $(x, y, l)$, have the local ion temperature $T_{i}(x)$. The probability that they escape without collisions with plasma particles onto the wall point $(0,0,0)$ is $\omega_{w}(x, y, l)=\exp (-\varepsilon / \cos \psi)$, where $\varepsilon=\int_{0}^{x} \frac{\nu_{i o n}+\nu_{*}}{V_{i}(x)} d x^{\prime}$. The density of the energy flux transported by hot atoms to the wall can be assessed as $q_{c x}^{w}=$ $\int_{0}^{\infty} d x \int_{-\infty}^{\infty} F_{c x}^{w} d l$ with

$$
F_{c x}^{w}(x, l)=\int_{-\infty}^{\infty} d y \frac{1.5 T_{i} k_{c x} n_{i}\left(n_{0}+n_{*}\right) \omega_{w}}{4 \pi\left(x^{2}+y^{2}+l^{2}\right)} \cos \psi .
$$

For an azimuthally symmetric cloud of hot atoms, with $\lambda_{*}=l_{*}$, one can get

$$
q_{c x}^{w}=0.75 \int_{0}^{\infty} T_{i} k_{c x} n_{i}\left[\eta_{0} \phi\left(\frac{x}{\lambda_{0}}\right)+\eta_{*} \phi\left(\frac{x}{\lambda_{*}}\right)\right] d x
$$

where $\phi(\xi)=\int_{1}^{\infty} \frac{\exp \left[-\xi^{2}(\varsigma-1)-\varepsilon \sqrt{\varsigma}\right]}{2 \varsigma^{1.5}} d \varsigma$. For rough estimates, the function $\phi$ can be evaluated by the pass method, provid$\operatorname{ing} \phi(\xi) \approx \exp (-\varepsilon) /\left(2 \xi^{2}+\varepsilon+1\right)$. 
Calculations have been done for the conditions of gas puff through the ITER first wall placed at the distance $d_{s w}$ from the separatrix positioned at $r_{s}=2 \mathrm{~m}$. The radial profiles of plasma parameters in the confined region inside separatrix are approximated by the following analytical relations:in the plasma core, $0 \leq r \leq r_{b} \equiv r_{s}-\Delta_{b}$ :

$$
\begin{aligned}
n_{e, i} & =n_{e, i}(0)-\left[n_{e, i}(0)-n_{e, i}\left(r_{b}\right)\right] \times r^{2} / r_{b}^{2}, \\
T_{e, i} & =T_{e, i}(0)-\left[T_{e, i}(0)-T_{e, i}\left(r_{b}\right)\right] \times r^{2} / r_{b}^{2}
\end{aligned}
$$

in the ETB with the width $\Delta_{b}, r_{b} \leq r \leq r_{s}$ :

$$
\begin{aligned}
n_{e, i} & =n_{e, i}\left(r_{b}\right)-\left[n_{e, i}\left(r_{b}\right)-n_{e, i}\left(r_{s}\right)\right] \times\left(r-r_{b}\right) / \Delta_{b}, \\
T_{e, i} & =T_{e, i}\left(r_{b}\right)-\left[T_{e, i}\left(r_{b}\right)-T_{e, i}\left(r_{s}\right)\right] \times\left(r-r_{b}\right) / \Delta_{b} .
\end{aligned}
$$

Here, $\Delta_{b}=0.04 \mathrm{~m}, n_{e}(0)=1.1 \cdot 10^{20} \mathrm{~m}^{-3}, n_{e}\left(r_{b}\right)=10^{20} \mathrm{~m}^{-3}$, $T_{e}(0)=30 \mathrm{keV}$ and $T_{e}\left(r_{b}\right)=3.2 \mathrm{keV}$ are taken according to
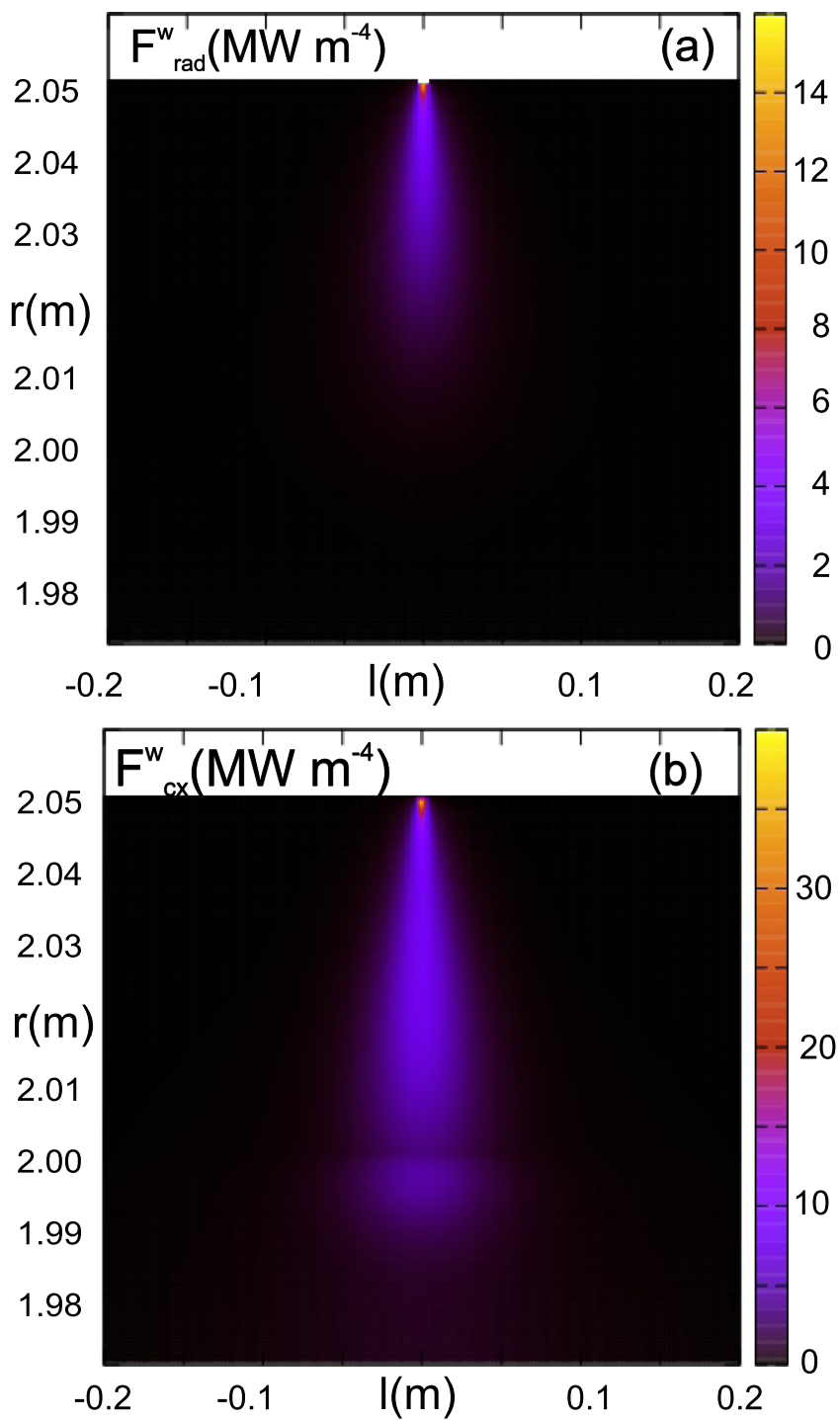

FIG. 3. Characteristics of the energy loss to the wall by deuterium gas puff with radiation (a) and charge-exchanged atoms (b) computed for the distance from the separatrix to the wall $d_{s w}$ of $0.05 \mathrm{~m}$ and the gas influx $\Phi_{0}=10^{22} \mathrm{~s}^{-1}$.
Ref. $14 ; n_{e}\left(r_{s}\right)=2.5 \times 10^{19} \mathrm{~m}^{-3}$ and $T_{e}\left(r_{s}\right)=0.22 \mathrm{keV}$ at the separatrix-Ref. 15. Within the SOL, the parameters are approximated as exponentially decaying

$$
\begin{aligned}
& n_{e}(r)=n_{e}\left(r_{s}\right) \exp \left[-\left(r-r_{s}\right) / \lambda_{n}\right], \\
& T_{e}(r)=T_{e}\left(r_{s}\right) \exp \left[-\left(r-r_{s}\right) / \lambda_{T}\right]
\end{aligned}
$$

with the $e$-folding lengths $\lambda_{n}=0.026 \mathrm{~m}$ and $\lambda_{T}=0.014 \mathrm{~m} ;{ }^{15}$ in addition $T_{i}=T_{e}$ is assumed and $n_{i}=0.95 n_{e}$ due to the plasma dilution with impurities. ${ }^{14}$ The gas puff takes place through the inlet with the area $S_{i n j}=0.001 \mathrm{~m}^{2}$ and the boundary conditions (11) are applied by describing primary atoms. Before the puff, the concentration of hydrogen isotopes in the wall is relatively small and neutrals generated by charge-exchange are efficiently absorbed by the wall, i.e., $R_{\text {rec }}=0$. Figure 3 demonstrates the energy loss characteristics $F_{r a d}$ and $F_{c x}$ as functions of the coordinates $x$ and $y$ calculated for $d_{s w}=0.05 \mathrm{~m}$ and the gas influx $\Phi_{0}=10^{22} \mathrm{~s}^{-1}$. The energy load densities on the wall with radiation, $q_{\text {rad }}^{w}$, and charge-exchanged atoms, $q_{c x}^{w}$, are shown in Figure 4 versus the distance $d_{s w}$ between the separatrix and the wall. Since any impact of puffed neutrals on the local plasma was neglected, the characteristics above are changing linearly with $\Phi_{0}$. One can see the heat load onto the wall induced by local puffing can exceed significantly an expected nominal value of $0.5 \mathrm{MW} \mathrm{m}{ }^{-2}$. ${ }^{16}$ This fact has to be taking into account by planning puffing systems. By an intensive enough puff, the energy losses and electron production in the neutral cloud may lead to the formation of a cold dense plasma bubble like multifaceted asymmetric radiation from the edge (MARFE), ${ }^{17}$ as it has been demonstrated for the case of impurity injection. ${ }^{2,9}$ The role of such bubbles for the heat loads onto the wall will be analyzed elsewhere.

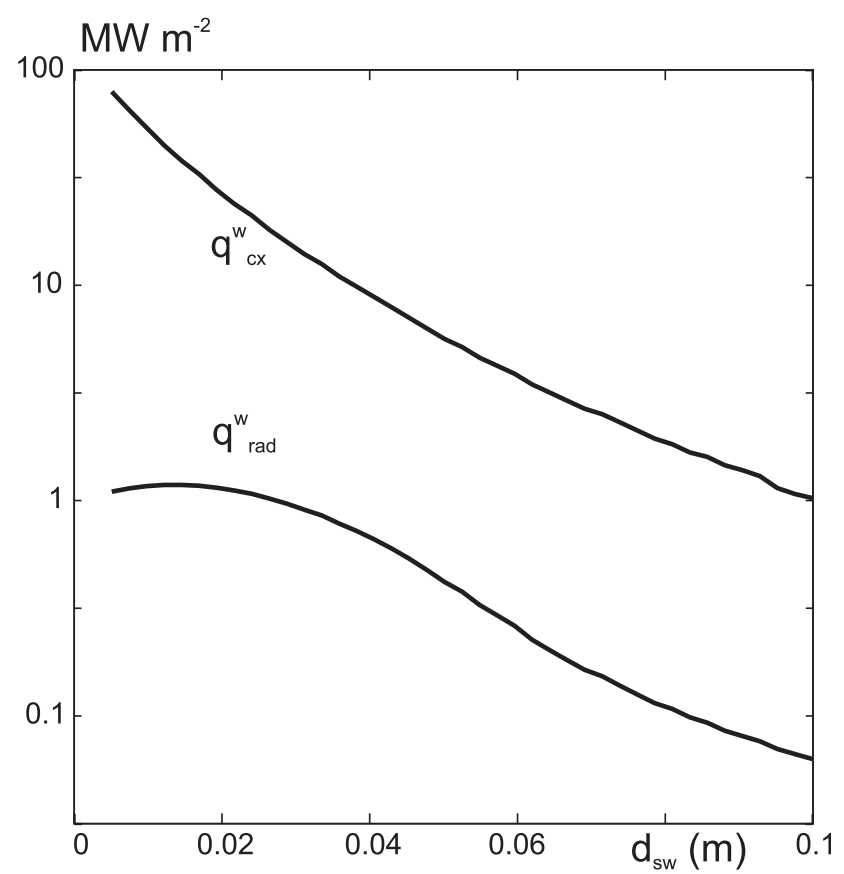

FIG. 4. The heat load density on the wall with radiation, $q_{\text {rad }}^{w}$, and charge-exchanged atoms, $q_{c x}^{w}$, versus the distance from the separatrix to the wall. 


\section{CONCLUSION}

Very localized injection of neutral particles of the working and impurity gases routinely takes place in fusion devices, both in deliberate and accident ways. For example, by using laser-based diagnostics, the particles resided in thin surface layer on the wall are released within a short intense bunch. In the plasma, they are excited by electrons, emit light and, by measuring this, one can assess the total number of particles ejected and, thus, the wall composition. Puffing of the working gas for the plasma density control is also done through inlets much smaller than the total wall area.

The non-stationarity and three-dimensionality of the neutral density profiles and inhomogeneity of plasma parameters require application of time demanding modeling tools. In the present paper, we elaborate an approach which allows to reduce the problem to solve the one-dimensional equations of the first/second order, describing the time evolution of the radial profiles of several characteristic parameters, namely, the maximum density of particles on the flux surfaces and dimensions of the regions occupied by diverse species on the surfaces. Both primary neutral particles, ejected into the plasma, and the secondary ones, generated, e.g., in charge-exchange collisions with the background ions, are included into the consideration. By necessity, the model can be extended to include other particle species, e.g., neutral molecules.

The approach elaborated is applied to describe the penetration of carbon atoms released into a TEXTOR deuterium plasma by a short laser pulse. The computations reproduce well the experimental observations, in particular, the time evolution of the radiation pattern of C-atoms with a particular wave length. Additionally, the puff of the main working gas into the ITER SOL plasma is considered to estimate the maximum heat loads onto the wall due to radiation of excited neutrals and outflow of hot atoms produced by charge-exchange. Calculations show that for typical puffing rates, heat loads on the wall may exceed significantly the nominal ones.

As the next step, the model has to be complemented with a consideration of the background plasma to take into account possible modifications induced by local cooling and generation of electrons through ionization of neutrals. Previous studies with the usage of essentially less sophisticated models for the neutral spreading process have shown that under stationary conditions, a significant increase in the electron density and decrease in the temperature can be expected if the impurity influx exceeds a critical level. It is of interest to study such phenomena under the conditions of ultra-short ejection of neutrals, e.g., by the application of laser-based diagnostics.

${ }^{1}$ V. Philipps, A. Malaquias, A. Hakola, J. Karhunen, G. Maddaluno, S. Almaviva, L. Caneve, F. Colao, E. Fortuna, P. Gasior, M. Kubkowska, A. Czarnecka, M. Laan, A. Lissovski, P. Paris, H. J. van der Meiden, P. Petersson, M. Rubel, A. Huber, M. Zlobinski, B. Schweer, N. Gierse, Q. Xiao, and G. Sergienko, Nucl. Fusion 53, 093002 (2013).

${ }^{2}$ M. Koltunov and M. Z. Tokar, Plasma Phys. Controlled Fusion 53, 065015 (2011)

${ }^{3}$ D. Reiter, M. Baelmans, and P. Börner, Fusion Sci. Technol. 47, 172 (2005), see http://www.ans.org/pubs/journals/fst/a_698.

${ }^{4}$ I. Konomi, T. Motohiro, T. Kobayashi, and T. Asaoka, Appl. Surf. Sci. 256, $4959(2010)$

${ }^{5}$ P. C. Stangeby, The Plasma Boundary of Magnetic Fusion Devices (Institute of Physics Publishing, Bristol and Philadelphia, 2000), pp. 277-357.

${ }^{6}$ M. Koltunov and M. Z. Tokar, Plasma Phys. Controlled Fusion 54, 025003 (2012).

${ }^{7}$ W. M. Stacey, Jr., Fusion Plasma Analysis (Interscience, New York, 1981), p. 116

${ }^{8}$ M. Z. Tokar, Plasma Phys. Controlled Fusion 35, 1119 (1993).

${ }^{9}$ M. Z. Tokar and M. Koltunov, Phys. Plasmas 19, 042502 (2012).

${ }^{10}$ H. Suno and T. Kato, At. Data Nucl. Data Tables 92, 407 (2006)

${ }^{11}$ N. Gierse, "Assessment of laser induced ablation spectroscopy (LIAS) as a method for quantitative in situ surface diagnostic in plasma environments," Ph.D. thesis (Universität zu Köln, Cologne, 2014).

${ }^{12}$ M. Z. Tokar and M. Koltunov, Nucl. Fusion 53, 093014 (2013).

${ }^{13}$ R. K. Janev, D. E. Post, W. D. Langer, K. Evans, D. B. Heifetz, and J. C. Weisheit, J. Nucl. Mater. 121, 10 (1984)

${ }^{14}$ T. Onujun and Y. Pianroj, Nucl. Fusion 49, 075003 (2009).

${ }^{15}$ A. S. Kukushkin, H. D. Pacher, V. Kotov, D. Reiter, D. Coster, and G. W. Pacher, Nucl. Fusion 47, 698 (2007).

${ }^{16}$ V. Kotov, A. Litnovsky, A. S. Kukushkin, D. Reiter, and A. Kirschner, J. Nucl. Mater. 390-391, 528 (2009).

${ }^{17}$ M. Z. Tokar, J. Rapp, D. Reiser, U. Samm, F. C. Schüller, G. Sergienko, and P. C. de Vries, J. Nucl. Mater. 266-269, 958 (1999). 